\title{
CT Scan Findings of Five Mildly Symptomatic COVID Patients from India
}

\author{
Sanjith Saseedharan, Vaijanti Kadam, Roopa Karanam and Paritosh Baghel \\ Department of Critical Care, S.L. Raheja Hospital - Fortis Associate, Mahim, Mumbai, Maharashtra, India
}

\begin{abstract}
The outbreak of the SARS-Cov-2 (Severe Acute Respiratory Syndrome - Coronavirus 2) viral pandemic which started at Wuhan China has spread to 200 countries and have resulted in a huge loss to mankind and the global economy. Computerised Tomographic (CT) scan may play an important role in the diagnosis and management of the COVID -19-the disease caused by the SARS-Cov-2. This modality might help in triaging this extremely contagious disease and thus help serve in reducing the outbreak. We present five cases that presented to the hospital with mild symptoms, where the CT scan findings were disproportionate to the symptoms thus leading to effective triage. This resulted in effective disposition in isolation ward and thus helped to protect the healthcare workers from contraction of the disease. This is especially important as the definitive diagnosis via RT-PCR (Reverse Transcriptase - Polymerase Chain Reaction) would take almost 24 hours in the present time in India.
\end{abstract}

Key Words: CT scan, COVID- 19, Triage, SARS-Cov-2.

How to cite this article: Saseedharan S, Kadam V, Karanam R, Baghel P. CT Scan Findings of Five Mildly Symptomatic COVID Patients from India. J Coll Physicians Surg Pak 2020; 30(JCPSPCR):CR48-CR49 https://doi.org/10.29271/jcpsp.2020.JCPSPCR.CR48.

The novel Corona virus (SARS-Cov-2) identified in December 2019 as an outbreak in Wuhan capital of central China has now alarmingly created a pandemic across borders with the WHO declaring it a global health emergency. Many countries including India are presently in the midst of this health crisis. ${ }^{1}$ The prime reason to reach epidemic proportion is because of non-restriction, non-isolation of asymptomatic or mildly symptomatic contacts leading to local transmission and hence epidemic spread. Thus it is important to safeguard people and specifically the workforce of healthcare workers and take measures to prevent the spread .The turnaround time for Reverse Transcriptase-Polymerase Chain Reaction based tests are still annoyingly dismal and the wait becomes painful for the healthcare workers who may get exposed to the secretions or aerosols of COVID-19 positive patients. Because this virus primarily involves the respiratory system an High Resolution Computerised Tomography scan (HRCT) of chest seems to be an effective way of screening, evaluation and follow up of these patients. ${ }^{2}$

Thus based on recent literature the hospital infection steering committee decided to perform a HRCT (chest) scan for every patient that presented even a single symptom of cough, fever or breathlessness (influenza like) coming from the community. All patients presenting to the hospital werescreened:

Correspondence to: Dr. Sanjith Saseedharan, Department of Critical Care, S.L.Raheja Hospital - Fortis Associate, Mahim, Mumbai-400059, Maharashtra, India

E-mail: docsanjith@rediffmail.com

Received: April 11, 2020; Revised: April 13, 2020;

Accepted: April 14, 2020

DOI: https://doi.org/10.29271/jcpsp.2020.JCPSPCR.CR48
1. If they had fever, sore throat, dry cough, myalgia, GI symptoms, anosmia or breathlessness?

2. If they had been in close contact to a COVID-19 positive patient in the last 14 days.

3. If they had foreign travel in the last 14 days.

If the answer to any of the questions were positive these patients were called COVID-19 suspects and a CT scan was done after explaining to the relatives. The cost of the CTscan were borne by the patient or relatives themselves.

At the time of writing this paper we had screened 52 patients and performed 12 scans. We report the findings of the HRCT(chest) scans(128 slice) reconstructed by using a medium and sharp reconstruction in supine position at full inspiration and expiration for the first five patients who presented to the hospital and on screening(via questionnaire mentioned above) were positive and thus qualified for a CT scan. All the five patients later turned out to be laboratory confirmed cases. (At that time, i.e. March $14^{\text {th }}$, the guidelines were not clear in India) and the tests were available in one hospital in Mumbai, India).All the HRCT (chest) scans were reviewed by a senior radiologist and all 5 images (Figures 1a-e) showed similar typical findings suggestive of viral pneumonitis with patchy areas of bilateral peripheral ground glass opacities, interlobular septal thickening scattered in both the lungs and fibro atelectatic segments seen in the lung bases. None of the patients showed any marked degree of pleural effusions in these scans. Some of them showed dense consolidation with air bronchogram pattern. There was no lymphadenopathy in any of the scans. These changes were very much similar to the scans in COVID-19 patients reported in recent papers from western literature. ${ }^{1,2}$ 


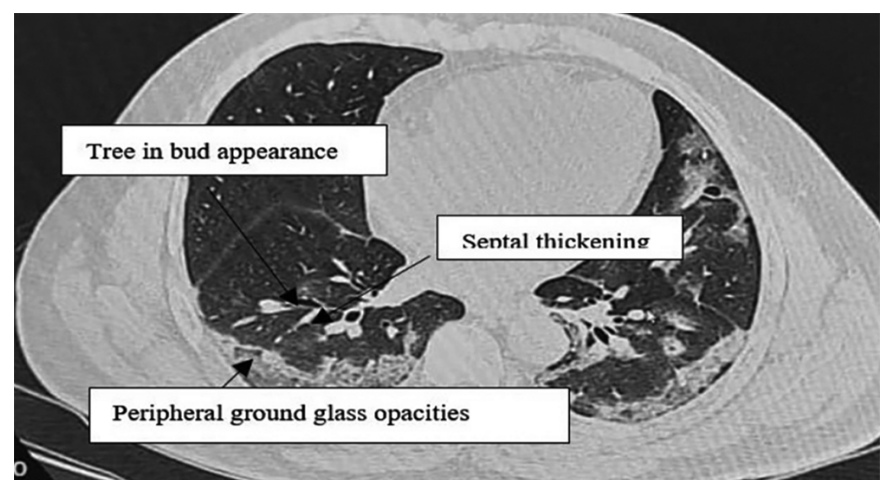

Figure 1 (a): 51-year man No comorbidities Complaints of (c/o) fever (100.4) for 1 day history of $(h / 0)$ of travel from Dubai 10 days back.

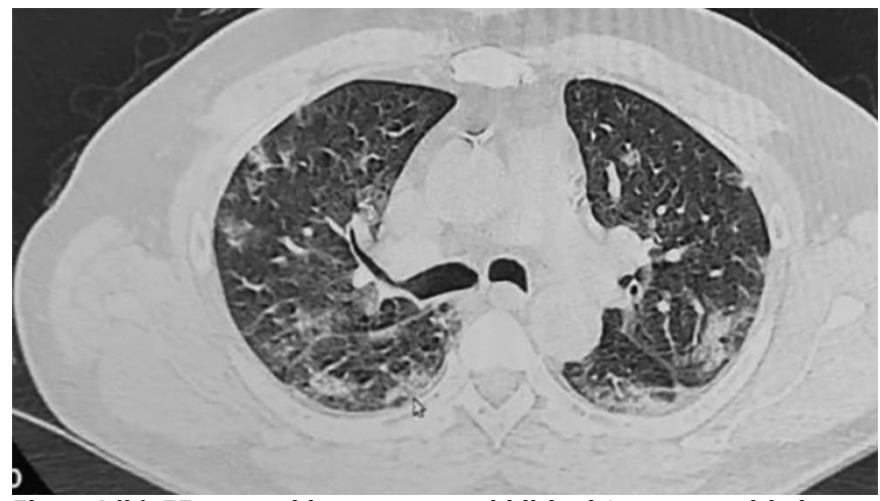

Figure1 (b): 55 -years old man no comorbidities h/o contact with the son who had arrived from London c/o mild sore throat.

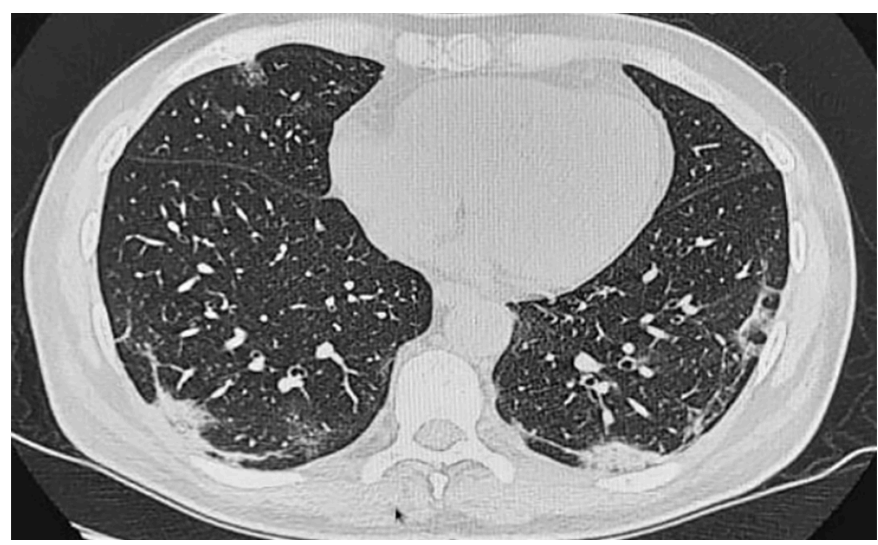

Figure 1 (c): 60 year old male doctor no comorbidity came with complaints h/o contact with Italian c/o malaise and sore throat.

Subsequently all the five patients were placed on the government advisory of hydroxy chloroquine $400 \mathrm{mg}$ twice on first day followed by $200 \mathrm{mg}$ twice a day for 5 days for mildly symptomatic patients along with Tab azithromycin $500 \mathrm{mg}$ for five days. All five patients are tested presently negative, have no symptoms and have been shifted out of the hospital and are presently in home isolation (14 more days) after 14 days of hospital isolation stay.

All these findings have the same spectrum as that described elsewhere. ${ }^{1,2}$ More importantly all these findings were seen when the patients were very mildly symptomatic and hence may help in screening out those patients for purposes of personal protection of healthcare workers and isolation of these patients to prevent spread of COVID-19.

These findings on CT scan would thus help the triage staff to understand how to tackle the COVID-19 epidemic which is likely to hit the region infull force in the nearfuture.

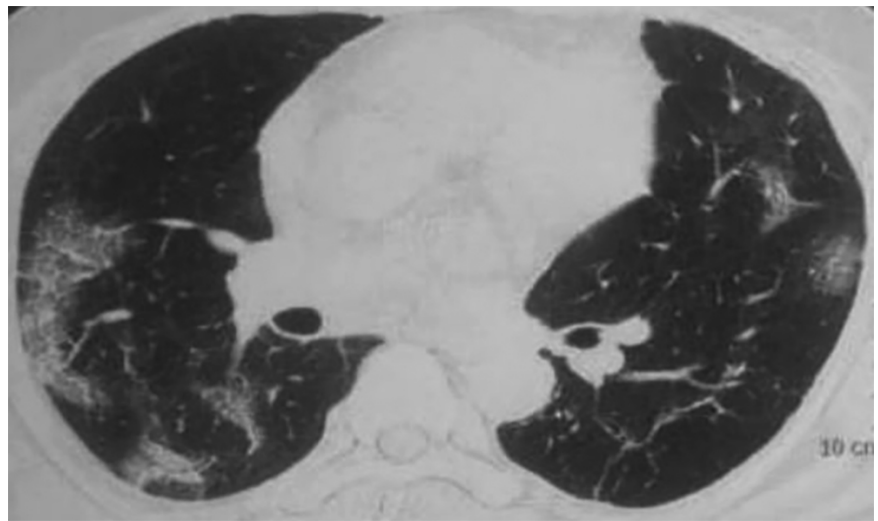

Figure 1 (d): 55-year man known hypertension no h/o contact with a positive patient c/odry cough.

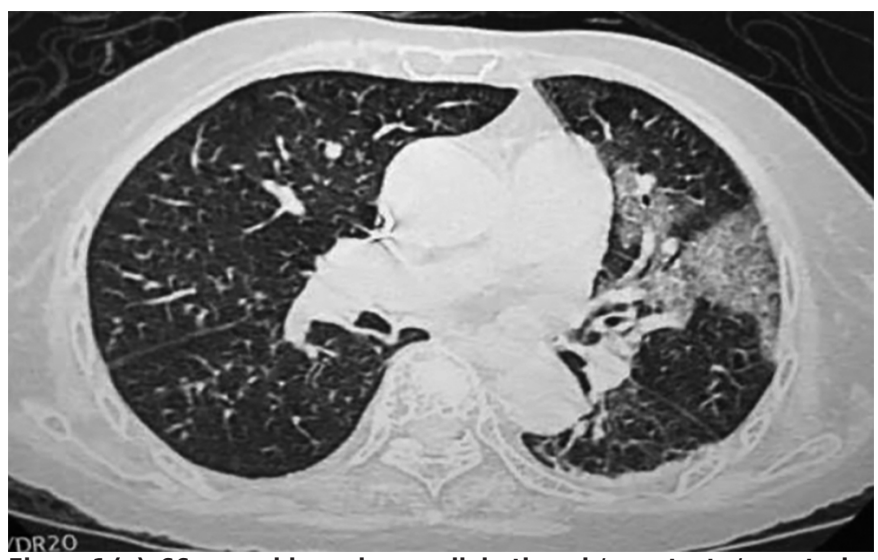

Figure 1 (e): 66-year old man known diabetic no h/o contact c/o gastrointestinal upset, mild fever.

\section{CONFLICT OF INTEREST:}

Authors declared no conflict of interest.

\section{AUTHORS' CONTRIBUTION:}

SS: Conceived the idea and wrote the manuscript.

VK: Literature search.

RK: Manuscriptediting.

PB: Collection of data.

\section{REFERENCES}

1. 1.Li Y, Xia L. Coronavirus Disease 2019 (COVID-19): Role of Chest CT in Diagnosis and Management. AJR Am J Roentgenol 2020; 4:1-7.

2. Jin YH, Cai L, Cheng ZS, Cheng H, Deng T, Fan YP, et al. A rapid advice guideline for the diagnosis and treatment of 2019 novel coronavirus (2019-nCoV) infected pneumonia (standard version). Mil Med Res 2020; 7(1):4. 